\title{
Death effector domain-containing proteins DEDD and FLAME-3 form nuclear complexes with the TFIIIC102 subunit of human transcription factor IIIC
}

\author{
Y Zhan ${ }^{1,2,3}$, R Hegde ${ }^{1,2,3}$, SM Srinivasula ${ }^{1,2,3}$, \\ $T_{\text {T Fernandes-Alnemri }}{ }^{1,2}$ and ES Alnemri ${ }^{\star, 1,2}$ \\ 1 Center for Apoptosis Research, Kimmel Cancer Institute, Thomas Jefferson \\ University, Philadelphia, PA 19107, USA \\ 2 Department of Microbiology and Immunology, Kimmel Cancer Institute, \\ Thomas Jefferson University, Philadelphia, PA 19107, USA \\ 3 These authors contributed equally to this work \\ * Corresponding author: ES Alnemri, Thomas Jefferson University, Kimmel \\ Cancer Institute, Bluemle Life Sciences Building, Room 904, 233 S 10th Street, \\ Philadelphia, PA 19107, USA. Tel: 215-503-4632; Fax: 215-923-1098; \\ E-mail: E_Alnemri@lac.jci.tju.edu
}

Received 8.2.02; accepted 8.2.02

Edited by G Melino

\begin{abstract}
Death effector domain-containing proteins are involved in important cellular processes such as death-receptor induced apoptosis, NF- $\kappa$ B activation and ERK activation. Here we report the identification of a novel nuclear DED-containing protein, FLAME-3. FLAME-3 shares significant sequence (46.6\% identical) and structural homology to another DEDcontaining protein, DEDD. FLAME-3 interacts with DEDD and c-FLIP (FLAME-1) but not with the other DED-containing proteins FADD, caspase-8 or caspase-10. FLAME-3 translocates to, and sequesters C-FLIP in the nucleus upon overexpression in human cell lines. Using the yeast twohybrid system to identify DEDD-interacting proteins, the TFIIIC102 subunit of human transcription factor TFIIIC was identified as a DEDD- and FLAME-3-specific interacting protein. Co-expression of either DEDD or FLAME-3 with hTFIIIC102 in MCF-7 cells induces the translocation from the cytoplasm and sequestration of hTFIIIC102 in the nucleus, indicating that DEDD and FLAME-3 form strong heterocomplexes with hTFIIIC102 and might be important regulators of the activity of the hTFIIIC transcriptional complex. Consistent with this, overexpression of DEDD or FLAME-3 in 293 cells inhibited the expression of a luciferase-reporter gene under the control of the NF- $k$ B promoter. Our data provide the first direct evidence for the involvement of DED-containing proteins in the regulation of components of the general transcription machinery in the nucleus.

Cell Death and Differentiation (2002) 9, 439-447. DOI: 10.1038/ sj/cdd/4401038
\end{abstract}

Keywords: Apoptosis; Death effector domain; TFIIIC; DEDD; FLAME-1; FLIP
Abbreviations: DED, death effector domain; EST, expressed sequence tags; CTD, C-terminal domain

\section{Introduction}

Death effector domain (DED)-containing proteins are important components of the cell death receptor-signaling pathway. ${ }^{1,2}$ Ligation of the death receptors, such as TNF, CD95 and TRAIL receptors, results in the recruitment of the adaptor molecule FADD/Mort-1 through homotypic interactions of its C-terminal death domain with the cytoplasmic death domain of the death receptors. ${ }^{1,3,4}$ FADD, which also contains an $\mathrm{N}$-terminal DED, transduces the activation signal from the death receptors to the DED-containing precursors (procaspases) of the initiator caspases 8 and 10, by direct interaction of its DED with their corresponding DEDs. ${ }^{1,5}$ The association of FADD with procaspase-8 and procaspase-10 results in their auto-processing/activation and the release of the mature caspases 8 and 10 from the membrane associated death receptor signaling complex into the cytoplasm. ${ }^{5-7}$ The mature caspase-8 and -10 could then cleave several important cellular substrates such as Bid and the effector caspases 3, 6 and 7 resulting in initiation of the caspase cascade and apoptosis. ${ }^{8,9}$ Thus, the DED regions of the adaptor molecule FADD and the initiator caspases 8 and 10 represent the link between the death receptors and the downstream cell death machinery.

Although some members of the family of DED-containing proteins, such as FADD, caspase- 8 and caspase-10, are agonists of the death receptor pathway, yet others, such as the viral and cellular FLIP proteins, are dominant negative inhibitors or antagonists to this pathway. ${ }^{10}$ The viral and cellular FLIP proteins inhibit the death receptor pathway by binding to the DED regions of FADD and/or caspases 8 and 10 preventing the formation of a productive death receptor signaling complex. ${ }^{10} \mathrm{C}$-FLIP-deficient mice do not survive, ${ }^{11}$ suggesting that the anti-apoptotic activity of cFLIP plays an important role in embryonic development.

Beside regulation of apoptosis, other important functions for DED-containing proteins have been reported. Several studies have shown that the DED protein PEA-15 is involved in the regulation of integrin activation, ERK activation, phospholipase D expression and glucose transport. ${ }^{12-15}$ Another DED protein, DEDD, was reported to bind to DNA, inhibit RNA polymerase I activity in vivo and inhibit transcription in an in vitro transcription assay. ${ }^{16,17}$ DED-containing proteins FADD, cFLIP, Caspase-8 and VFLIP can also activate NF- $\kappa$ B pathway through their DEDs. ${ }^{18-20}$ These findings indicate that DED-proteins are involved in diverse cellular processes ranging from cell survival to cell death. 
In order to identify novel cellular DED-containing and DED-interacting proteins, we searched the National Center for Biotechnology Information GenBank expressed-sequence tags (EST) database for sequences encoding uncharacterized DED-containing proteins related to DEDD. We report here the cloning and functional characterization of a previously uncharacterized DED-containing protein designated FLAME-3, which has high homology to DEDD. Using the yeast two-hybrid system we identified hTFIIIC102 subunit of TFIIIC transcription factor as DEDD- and FLAME-3- interacting protein. Consistent with this result we found that DEDD and FLAME-3 can sequester TFIIIC102 in the nuclei of transfected cells. Our results suggest that the interaction of DEDD and FLAME-3 with this subunit may regulate transcription of class III genes.

\section{Results}

\section{Cloning, sequence analysis and tissue distribution of FLAME-3}

Death effector domain (DED)-containing proteins play important roles in apoptosis and cellular signaling. ${ }^{1,10,21}$ To identify new DED-containing proteins, we searched the entire public GenBank ${ }^{T M}$ EST database for sequence homologus to previously known DED-containing proteins, using the tBLASTN program. One human EST clone (AA090354) encoding a partial open reading frame with significant homology to the C-terminal domain of DEDD protein was identified. Based on its sequence, PCR primers were generated and $5^{\prime}$-RACE was performed with a human PBL library. This resulted in cloning of the entire open reading frame of this new protein, which was subsequently named FLAME-3 based on its overall sequence and structural homology with DEDD/FLAME-2. The human FLAME-3 (Figure $1 \mathrm{~A}$ ) is $46.6 \%$ identical to DEDD and its domain structure is very similar to that of DEDD (Figure 1B). FLAME-3 has an N-terminal death effector domain (DED, residues $25-102)$, which is very similar to the DED domains present in other DED proteins such as DEDD, FADD, caspase-8, caspase-10 and c-FLIP (FLAME-1) (Figure 1C). Following the DED, FLAME-3 contains three central nuclear localization sequences (NLSs, residues 104-109, 131-134 and 153-174) and a C-terminal domain (CTD, residues 175-326), which has no significant homology to any known protein except DEDD.

The mouse FLAME-3 was also identified by searching the GenBank ${ }^{T M}$ EST database and a cDNA encoding its entire open reading frame was obtained by overlapping PCR. The human and mouse FLAME-3 proteins share $91 \%$ identity with each other (Figure 1A). Interestingly, most of the amino acid substitutions in the human and mouse sequences are present in the linker region, which contain the NLSs between the DED and CTD. The lack of significant substitutions in the sequences of the DED and CTD suggests that the overall structures of these domains are critical for the function of FLAME-3.

A BLAST search of the NCBI high-throughput genome sequences revealed that the human FLAME-3 gene is located on chromosome 19.

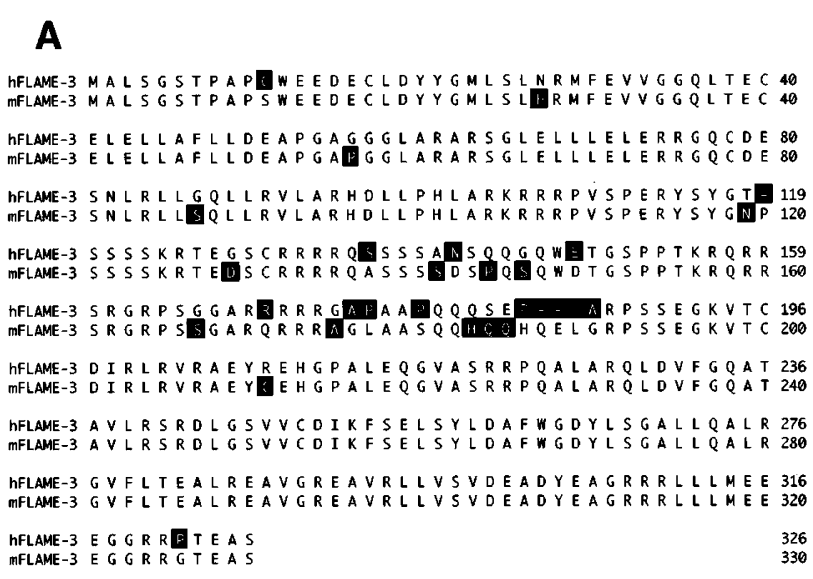

B
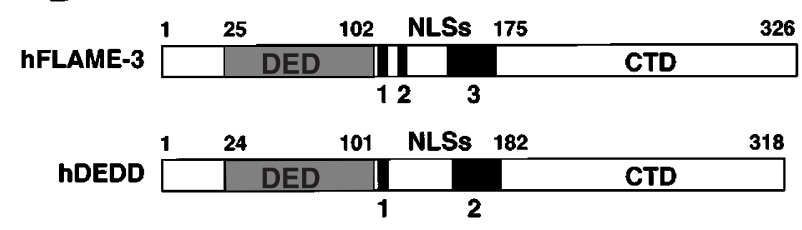

C

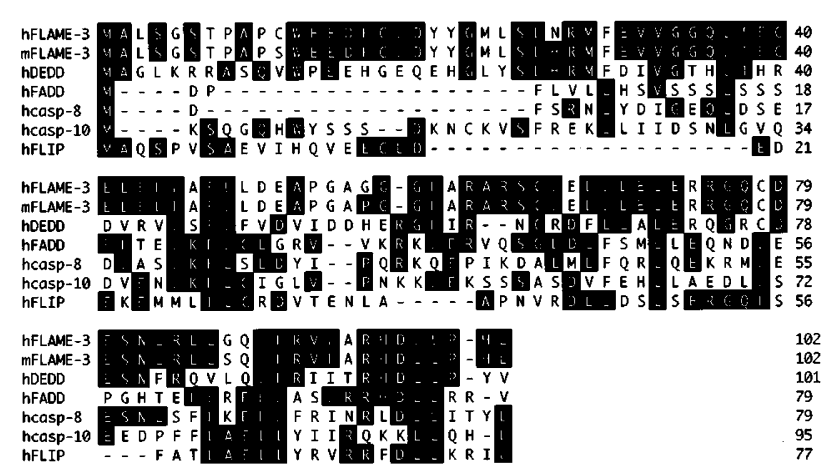

Figure 1 Sequence alignment and domain structure of FLAME-3. (A) Colinear alignment of the predicted amino acid sequences of human and mouse FLAME-3 proteins. Non-identical residues in the two sequences are shaded black. (B) Bar diagrams representing the domain structure of human FLAME-3 and DEDD proteins. The three NLSs in FLAME-3 (residues 104-109, 131134 and 153-174) and two NLSs in DEDD (residues 104-107 and 164-181) are indicated. (C) Amino acid sequence alignment of the DED of FLAME-3 with DEDs found in human DEDD, FADD, caspase-8, caspase-10 and c-FLIP. Black shading indicates identical residues

Northern blot analysis of multiple human tissues detected two FLAME-3 mRNA species of 1.6 and 2.0 kilobase pairs $(\mathrm{kb})$ in all tissues examined (Figure 2A). These two mRNA species could result from the use of alternative polyadenylation sites and/or alternative splicing of the FLAME-3 pre-mRNA. The highest level of expression of the FLAME-3 mRNA was found in peripheral blood leukocytes, spleen and testes and lower expression levels were detected in all other tissues tested. The high expression of FLAME-3 mRNA in peripheral blood leukocytes, spleen and testes is reminiscent of that of the DEDD mRNA, ${ }^{22}$ suggesting that the two genes are under 
A

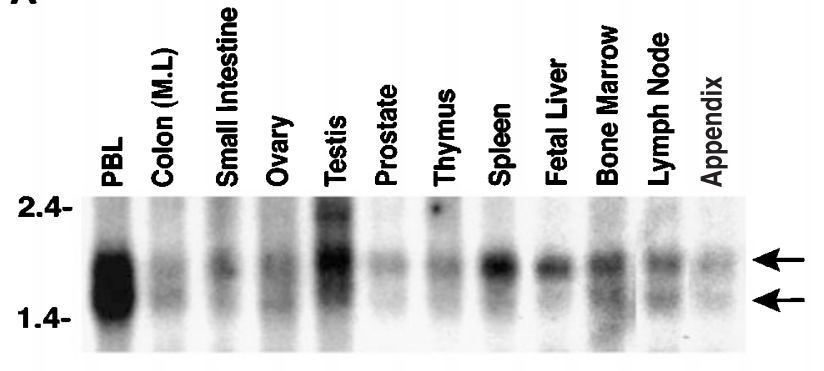

B

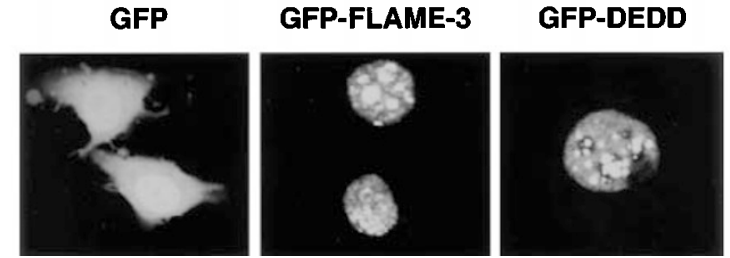

Figure 2 Tissue distribution and subcellular localization of FLAME-3. (A) Tissue distribution pattern of the human FLAME-3 mRNA. The Northern blot analysis was performed as described under Materials and Methods. The two major FLAME-3 mRNAs are indicated by arrows. PBL, peripheral blood leukocytes. (B) Subcellular localization of GFP-DEDD and GFP-FLAME-3. MCF-7 cells were transfected with $1 \mathrm{lg}$ of expression constructs encoding GFP, GFP-DEDD or GFP-FLAME-3 and $24 \mathrm{~h}$ later the cells were fixed and examined under a confocal laser scanning microscope

similar transcriptional regulation and their functions are important in these tissues.

\section{DEDD and FLAME-3 are nuclear proteins}

Given that DEDD and FLAME-3 contain typical NLSs between the DED and CTD regions, we tested whether transfection of cells with constructs encoding GFP-tagged DEDD or FLAME-3 would result in targeting of these proteins to the nucleus. As shown in Figure 2B both GFP - DEDD and GFP-FLAME-3 exclusively localized to the nuclei of the transfected cells. Of note, the two proteins accumulated in subnuclear structures resembling nucleoli, suggesting that these proteins may have a role in nucleolar functions.

\section{Interactions of FLAME-3 with other DED-containing proteins}

To investigate whether FLAME-3 can interact with other DEDcontaining molecules, which are involved in the death receptors pathway, we performed co-immunoprecipitation experiments. We co-transfected Flag-tagged FLAME-3 with different T7-tagged DED-containing molecules as indicated in Figure 3A. These experiments showed that FLAME-3 interacts with c-FLIP/FLAME-1 and DEDD, and weakly with caspase-8, but not with caspase-10 or the death receptors adaptor FADD.

To confirm these results we performed in vitro GST pulldown experiments with GST-tagged DEDD or FLAME-3 and different ${ }^{35} \mathrm{~S}$-labeled DED-containing proteins. Consistent with the co-immunoprecipitation results, neither GST-
DEDD nor GST - FLAME-3 fusion proteins did interact with FADD or caspase-10 (Figure 3B). However, both fusion proteins interacted with c-FLIP and weakly with caspase-8. The in vitro interaction experiments also revealed that DEDD and FLAME-3 proteins could homodimerize and heterodimerized with each other as evident from the ability of the GST-DEDD and GST-FLAME-3 to precipitate the ${ }^{35}$ S-labeled DEDD and FLAME-3 proteins (Figure 3B).

The ability of FLAME-3 to co-immunoprecipitate c-FLIP from cellular extracts expressing the two proteins prompted us to test if the two proteins can indeed interact with each other in vivo. To do this we transfected MCF-7 cells with constructs encoding a GFP-tagged c-FLIP and a Flagtagged FLAME-3 and then visualized the transfected cells by microscopy to see whether FLAME-3 could sequester c-FLIP in the nucleus. Given that FLAME-3 is a nuclear protein and C-FLIP is a cytoplasmic protein we expected to see the GFP-tagged c-FLIP in the nucleus if the two proteins interact with each other in vivo. As expected, coexpression of GFP-C-FLIP with FLAME-3 changed its cytoplasmic localization into nucleolar-like structures (Figure $3 \mathrm{C}$ ). These results indicated that the two proteins do indeed interact in vivo with each other.

Taken together these data suggest that FLAME-3, and perhaps DEDD, function distal to components of the death receptor pathway but could interact with and influence the subcellular localization of c-FLIP when overexpressed in mammalian cells.

\section{Identification of TFIIIC102 as DEDD-interacting protein}

The exclusive localization of DEDD and FLAME-3 in the nucleus suggests that these proteins could play important roles in nuclear functions. To study the potential role of DEDD proteins in the nucleus we decided to use the yeast twohybrid system to identify proteins that interact with the full length DEDD. A yeast expression vector was constructed by fusing the cDNAs of the Lex A DNA binding domain to the Nterminus of DEDD in-frame. This bait plasmid was cotransformed into yeast cells with a human brain cDNA library fused to a cDNA cassette encoding the SV40 nuclear localization sequence, the acid Blob B42, and the hemagglutinin epitope tag under the control of the yeast GAL-1 inducible promotor. Out of the 11 positive clones obtained, two clones exhibited strong blue $\beta$-Gal staining when cotransformed into yeast cells with the DEDD-bait plasmid but not with the empty bait plasmid (data not shown). Sequence analysis of the two clones revealed that they encode a short isoform of the TFIIIC102 subunit of human TFIIIC transcription factor. The TFIIIC102 short isoform (TFIIIC 102-s) is 413amino acid long as opposed to the 886-amino acid long fulllength human TFIIIC102 (hTFIIIC102) protein (Figure 4A and ref ${ }^{23}$ ). The short isoform is identical to the full length hTFIIIC102 in the first $406 \mathrm{~N}$-terminal amino acid sequence, and differs only in the last seven C-terminal amino acid sequence from the full-length hTFIIIC102. The mRNA encoding the short isoform of hTFIIIC102 contains an independent stop codon and an unmatched untranslated $3^{\prime}$ region with poly A tail (Genbank accession \# AF465407) 


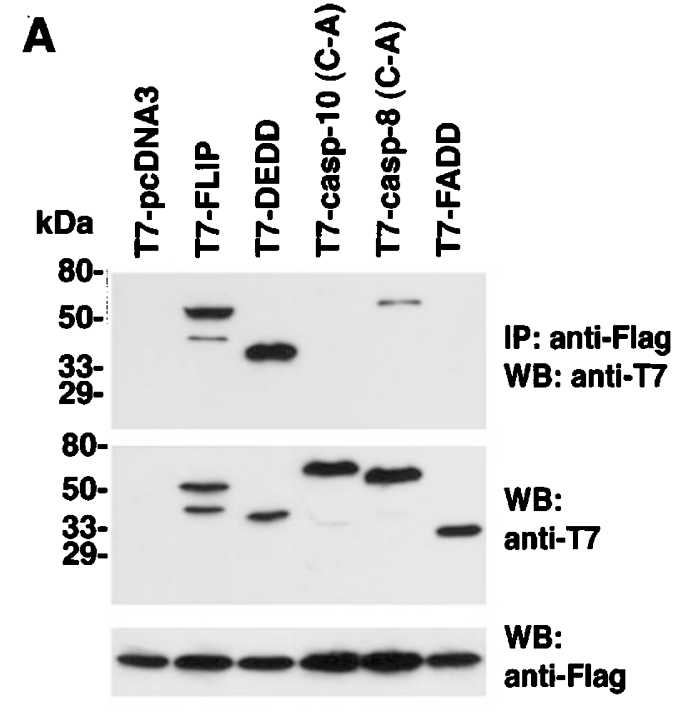

C

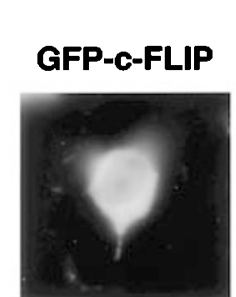

$$
\text { GFP-C-FLIP }
$$

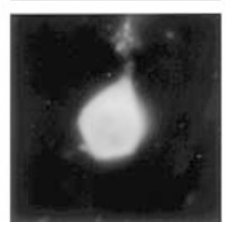
+ FLAME-3

GFP-FLAME-3
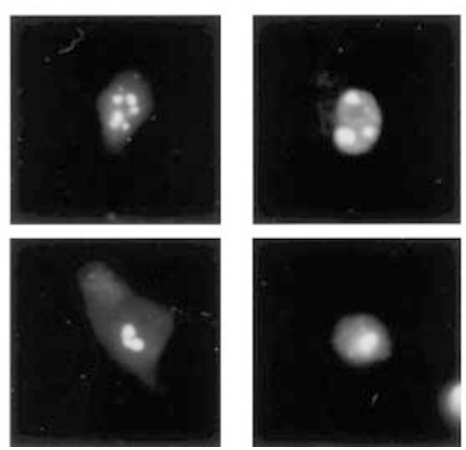

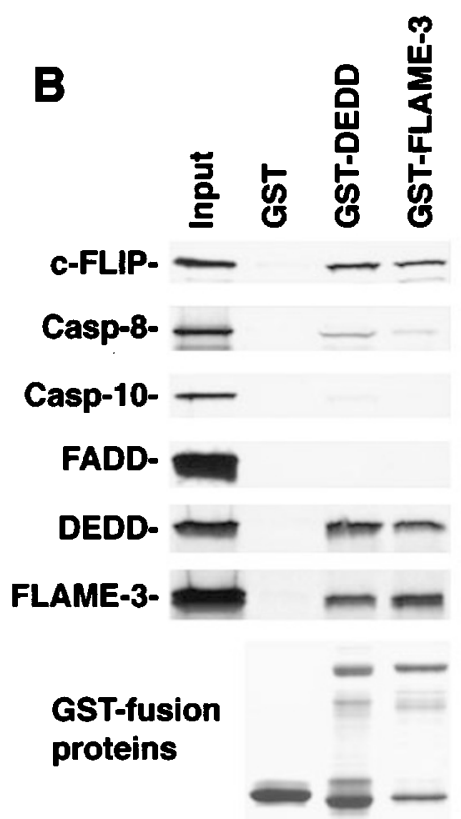

Figure 3 Interactions of FLAME-3 with other DED-containing proteins. (A) 293T cells were co-transfected with expression constructs encoding Flag-tagged FLAME-3 and the indicated T7-tagged molecules. Twenty-four hours after transfection, cells were lysed, and the lysates immunoprecipitated with Flag antibody. The immunoprecipitates were immunoblotted with anti T7 antibody (upper panel). The total cell lysates were also immunoblotted with either T7 (middle panel) or anti-Flag (lower panel) antibodies. (B) In vitro interaction of DEDD and FLAME-3 with other DED-containing proteins. GST, GST-DEDD or GST-FLAME-3 proteins bound to gluthatione-Sepharose beads were incubated with in vitro translated ${ }^{35}$ S-labeled c-FLIP, caspase-8, caspase-10, FADD, DEDD or FLAME-3. The interactions were analyzed by SDS-PAGE and autoradiography. A Coomassie stained gel of the GST-fusion proteins is shown at the bottom. (C) Overexpression of FLAME-3 changes the subcellular localization of C-FLIP. MCF-7 cells were transfected with constructs encoding GFP-c-FLIP, GFP-C-FLIP plus T7-FLAME-3, GFP-FLAME-3, GFP or GFP plus T7-FLAME-3 as indicated. After $24 \mathrm{~h}$ of transfection, cells were fixed and viewed (100 $\times$ ) using a fluorescence microscope. Data are representative of at least three individual experiments $(n=3)$

which appears to be the result of the use of alternative splicing and polyadenylation sites in the hTFIIIC102 pre-mRNA.

To rule out that the short isoform is a cloning artifact, we generated sense and antisense primers, where the sense primer corresponds to an identical sequence in the two hTFIIIC102 isoforms and the antisense primer corresponds to a region specific only to the short isoform $3^{\prime}$ untranslated region. The two primers were used to perform a PCR on a human Jurkat cDNA library as a template. The generated PCR products were cloned and sequenced and found to be identical to the CDNA of the hTFIIIC102 short isoform isolated from the human Brain cDNA library by the yeast two-hybrid method.

Since there was no published information on the tissue distribution of hTFIIIC102, ${ }^{23}$ we performed Northern blot analysis of multiple human tissues and cancer cell lines using a probe from the coding region of the hTFIIIC102. Figure 4B shows three major transcripts of different sizes ( 3.2, 2.5 and 1.2) expressed mainly in the testes, heart and skeletal muscle. The heart contains predominantly the 


\section{A}

1 MSGFSPELIDYLEGKISFEEFERRREERKTREKKSLQEKGKLSAEENPDD 51 SEVPSSSGINSTKSQDKDVNEGETSDGVRKSVHKVFASMLGENEDDEEEE 101 EEEEEEEEEEETPEQPTAGDVFVLEMVLNRETKKMMKEKRPRSKLPRALR 151 GLMGEANIRFARGEREEAILMCMEI IRQAPLAYEPFSTLAMIYEDQGDME 201 KSLQFELIAAHLNPSDTEEWVRLAEMSLEQDNIKQAIFCYTKALKYEPTN 251 VRYLWERSSLYEQMGDHKMAMDGYRRILNLLSPSDGERFMQLARDMAKSY 301 YEANDVTSAINI IDEAFSKHOGLVSMEDVNIAAELY ISNKOYDKALEI IT 351 DFSGIVLEKKTSEEGTSEENKAPENVTCTIPDGVPIDITVKIMVCLVHLN 401 ILEPLNVCNKICT*

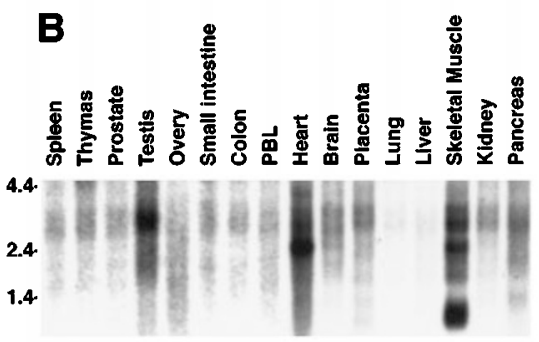

Normal tissues

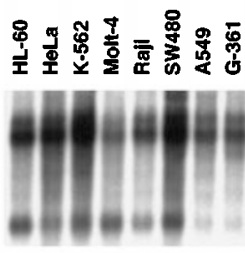

Cancer cell lines

Figure 4 Amino acid sequence of TFIIIC102-s and tissue distribution of hTFIIIC102. (A) Predicted amino acid sequence of the short isoform of hTFIIIC102 identified as a DEDD-interacting protein by the yeast two-hybrid method. The last seven residues that do not correspond to residues in the full length hTFIIIC102 are boxed. (B) Tissue distribution pattern of the human hTFIIIC102 mRNA in human normal tissues and tumor cell lines. The Northern blot analysis was performed as described under Materials and Methods. HL60, Promyelocytic leukemia; HeLa, HeLa cell S3; K-562, chronic myelogenous leukemia; MOLT-4, lymphoblastic leukemia; Raji, Burkitt's lymphoma; SW480, colorectal adenocarcinoma; A549, lung carcinoma; G361, melanoma. Numbers on the left indicate kilobases. PBL, peripheral blood leukocyte

$2.5 \mathrm{~kb}$ transcript, and skeletal muscle has an additional $1.2 \mathrm{~kb}$ major transcript. In testis, only the $3.2 \mathrm{~kb}$ transcript is observed. These varied transcript sizes indicate tissue specific expression of different isoforms of the protein. The cancer cell lines invariably contain three major transcripts corresponding to $3.5,3.2$ and $1.2 \mathrm{~kb}$. Of note, the $3.2 \mathrm{~kb}$ transcript is upregulated in most of the tumor cell lines, suggesting possible involvement in transformation.

\section{DEDD and FLAME-3 interact with hTFIIIC102 in vitro and in vivo}

We next investigated the interaction of DEDD and FLAME-3 with hTFIIIC102 and hTFIIIC102 short isoform by coimmunoprecipitation and GST-pull down experiments. As expected, both hTFIIIC102 and hTFIIIC102 short isoform were precipitated by DEDD and FLAME-3 proteins (Figure $5 A, B)$, indicating that not only DEDD but also FLAME-3 could interact with hTFIIIC102 and its short isoform.

To examine the interactions of DEDD proteins with hTFIIIC102 in vivo we transfected MCF7-Fas cells with constructs encoding GFP-tagged hTFIIIC102 or hTFIIIC102 short isoform, together with constructs encoding Flagtagged DEDD or FLAME-3. The transfected cells were then monitored by fluorescent confocal laser scanning microscopy (Figure 5C). Both GFP-hTFIIIC102 and GFPhTFIIIC102 short isoform were largely in the cytoplasm. However, in the presence of co-expressed DEDD or
$\mathbf{A}$

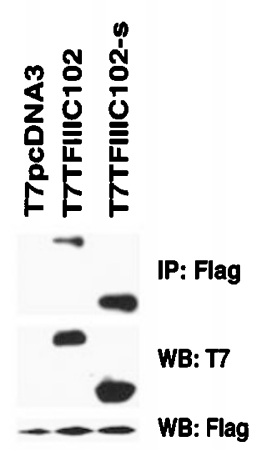

$\mathbf{B}$

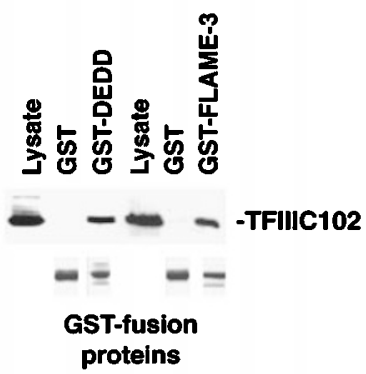

C

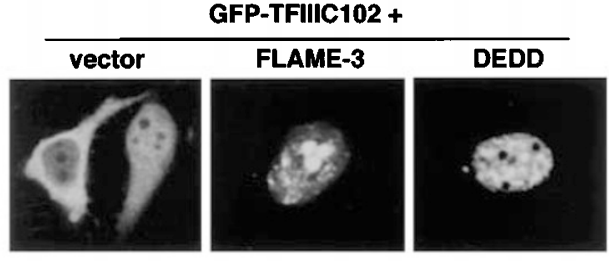

GFP-TFIIC102-8 +

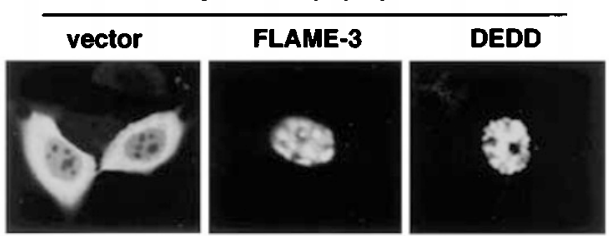

GFP +

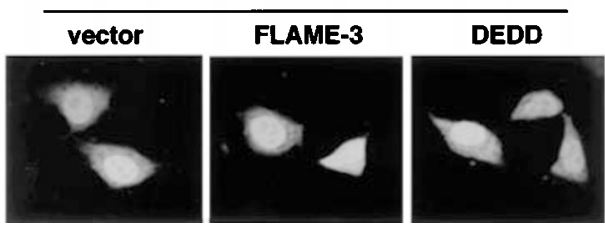

Figure 5 In vivo and in vitro interaction of DEDD and FLAME-3 proteins with hTFIIIC102. (A) 293T cells were co-transfected with expression constructs encoding Flag-tagged FLAME-3 and T7-tagged TFIIIC102 or TFIIIC102-s. Twenty-four hours after transfection, cells were lysed, and the lysates immunoprecipitated with Flag antibody. The immunoprecipitates were immunoblotted with anti T7 antibody (upper panel). The total cell lysates were also immunoblotted with either T7 (middle panel) or anti-Flag (lower panel) antibodies. (B) 293T cells were transfected with an expression plasmid encoding T7-tagged TFIIIC102 and $36 \mathrm{~h}$ after transfection, cells were lysed, and the lysates precipitated with either GST, GST - DEDD or GST - FLAME-3. The pellets and corresponding cellular lysates were analysed on SDS-PAGE and immunodetected with horse-radish peroxidase-conjugated T7 antibody. A Coomassie stained gel of the GST-fusion proteins is shown at the bottom. (C) Overexpression of DEDD or FLAME-3 sequesters TFIIIC102 and TFIIIC102-S in the nucleus. MCF-7 cells were transfected with constructs encoding GFP. TFIIIC102 (upper panels), GFP-TFIIIC102-s (middle panel) or GFP (Lower panel) together with empty vector (left panels) or constructs encoding T7tagged DEDD (right panels) or FLAME-3 (middle panels). After $24 \mathrm{~h}$ of transfection, cells were fixed and viewed using a confocal laser scanning microscope. Data are representative of at least three individual experiments $(n=3)$

FLAME-3 both GFP-hTFIIIC102 and GFP-hTFIIIC102 short isoform were sequestered in the nucleus in neucleoli-like structures similar to those observed with GFP-DEDD or GFP-FLAME-3. These observations provide clear evi- 
dence that the DEDD proteins interact with hTFIIIC102 in cells and may regulate its transport from the cytoplasm to the nucleus under physiological circumstances.

\section{Overexpression of DEDD and FLAME-3 inhibits NF- $\kappa$ B-regulated gene expression}

hTFIIIC is a multi-subunit general transcription complex that mediates transcription of class III genes. ${ }^{23-25}$ hTFIIIC directly recognizes promoters for tRNA and virus-associated RNA genes or promoter-TFIIIA complexes and subsequently recruits TFIIIB and RNA polymerase III to this complex. ${ }^{24,25}$ Given that DEDD and FLAME-3 can bind and sequester TFIIIC102 in the nucleus, we asked whether their overexpression in human cell lines could interfere with the general transcription machinery. To test this hypothesis, we utilized the NF- $\kappa$ B-luciferase reporter plasmid to measure the level of luciferase activity in cells treated with TNF- $\alpha$ in the presence or absence of overexpressed DEDD or FLAME-3. As shown in Figure 6, TNF- $\alpha$ treatment of the vector transfected control cells resulted in fivefold induction of luciferase activity compared to twofold in the DEDD or FLAME-3-expressing cells. These results indicate that overexpression of DEDD and FLAME-3 inhibits the expression of NF- $\kappa$ B-regulated genes, perhaps by sequestering TFIIIC102, resulting in a decrease in the activity of the TFIIIC complex. Overexpression of DEDD or FLAME-3 was also able to inhibit yeast GAL-4-induced luciferase activity in 293 cells, as determined with a GAL-4luciferase reporter plasmid (data not shown). Combined, our data indicate that overexpression of DEDD or FLAME-3 inhibits the general transcription machinery.

The ability of DEDD and FLAME-3 to inhibit NF- $\kappa$ Bregulated gene expression might be responsible for the weak apoptotic activity observed in two different studies

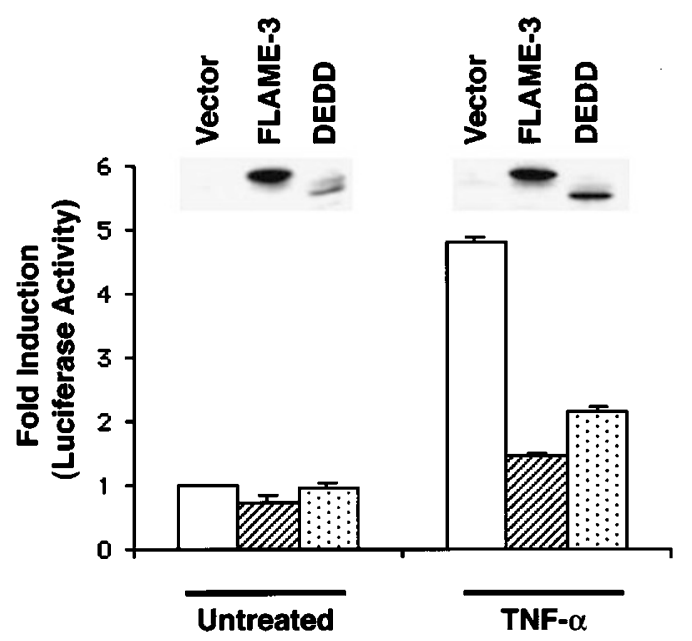

Figure 6 FLAME-3 and DEDD inhibit NF- $\kappa$ B-regulated transcription. A, 293 cells were transfected with $5 \times \mathrm{NF}-\kappa \mathrm{B}$-Luciferase reporter together with either empty vector or expression constructs for Flag-DEDD or Flag-FLAME-3. Twenty-four hours after transfection, cells were either left untreated or incubated with TNF- $\alpha$ for $5 \mathrm{~h}$. Cells were then collected and lysed, and the luciferase activity in the cell lysates was determined. pRSC-LacZ was included in all transfection reactions to normalize the transfection efficiency. The data represents three different experiments with overexpressed DEDD and DEDD-2/FLAME-3. ${ }^{16,26}$ However, in our hands we were unable to detect significant apoptosis induction or caspase activation by the overexpressed FLAME-3 compared to other apoptotic proteins such as FADD or caspase-8 (Figure 7A,B). The FLAME-3expressing cells had only $10 \%$ more apoptosis than the control vector-transfected cells $72 \mathrm{~h}$ after transfection. FLAME-3 and DEDD were also unable to significantly potentiate cell death by Fas, TRAIL or TNF (Figure 7C). This indicates that the primary function of the DEDD proteins might not be the regulation of apoptosis per se but most probably they are involved in the regulation of general transcription. This is consistent with a recent finding that DEDD inhibits RNA polymerase I dependent transcription and its weak apoptotic activity is dependent on its localization to the nucleus. ${ }^{17}$ Targeting of the DED region of DEDD-2/FLAME-3 was also found to be necessary for its weak pro-apoptotic activity. ${ }^{26}$ Taken together, these ob-
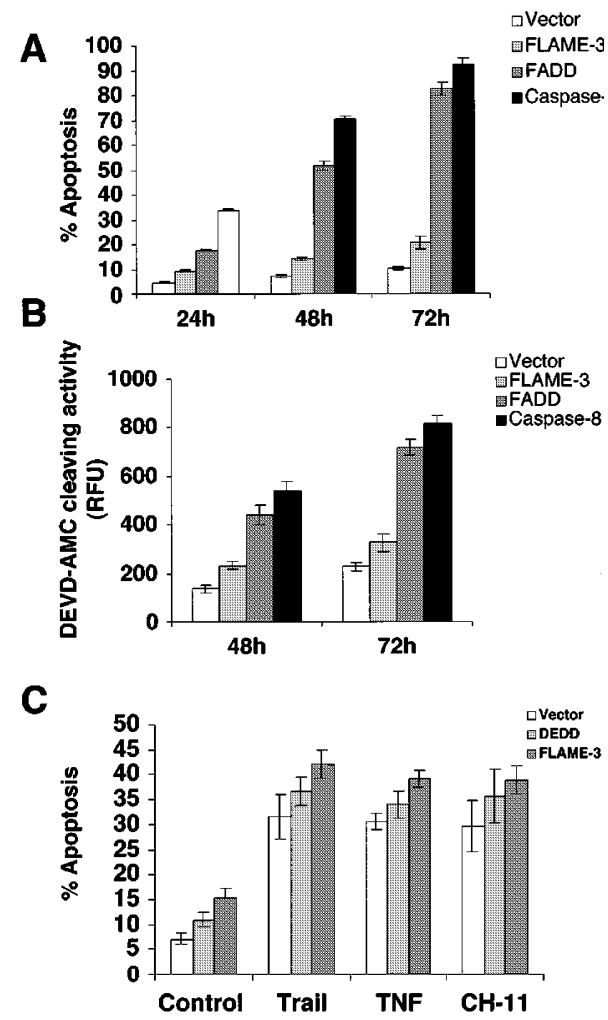

Figure 7 (A) Apoptotic activity of FLAME-3 compared with FADD and caspase-8. MCF-7-Fas cells were transfected with empty vector or expression constructs encoding FLAME-3, FADD or caspase-8 together with a GFPreporter plasmid. The percentages of apoptotic cells were determined after 24 , 48 and $72 \mathrm{~h}$ after transfection as described in the Materials and Methods. (B) 293 cells were transfected with empty vector or expression constructs encoding FLAME-3, FADD or caspase-8 and the cells were lysed 48 and $72 \mathrm{~h}$ after transfection. The DEVD-AMC cleaving activities of the cellular extracts were determined with the fluorogenic substrate DEVD-AMC $(50 \mu \mathrm{M})$ by fluorometry. (C) MCF-7-Fas cells were transfected with the indicated expression constructs (vector, DEDD, FLAME-3). Twenty-four hours after transfection the cells were left untreated (control) or treated with TRAIL, TNF- $\alpha$ or the agonist anti-Fas antibody $\mathrm{CH}-11$ as indicated for $5 \mathrm{~h}$. The percentage of round apoptotic cells was determined as described under Materials and Methods 
servations suggest that the overexpressed DEDD or FLAME-3 proteins could induce apoptosis from within the nucleus perhaps by sequestering important components of the general transcription machinery such as hTFIIIC102. Therefore, we believe that the inhibition of the RNA polymerase $\mathrm{I}^{-17}$ and RNA polymerase III- (a component of the TFIIIC complex) dependent transcription by the overexpressed DEDD or FLAME-3 proteins might be responsible for the observed inhibition of the NF- $\kappa$ B-regulated gene expression (Figure 6). This is expected to have serious consequences on cell survival and could ultimately lead to cell death.

\section{Discussion}

We have identified and characterized a novel nuclear DEDcontaining protein, FLAME-3, and its interacting proteins cFLIP and hTFIIIC102. FLAME-3 contains an N-terminal DED region, three central NLSs and a C-terminal domain highly related to that of DEDD. Based on Northern blot analysis, FLAME-3 mRNA is expressed mainly in PBL, spleen and testis, a distribution that is very similar to that of DEDD. Of note, FLAME-3 can heterodimerize with DEDD and thus these proteins could function as heterodimers in these tissues. FLAME-3 and DEDD can interact with c-FLIP and to a lesser extent with caspase-8, but not with FADD or caspase-10, suggesting that the DEDD and FLAME-3 proteins function distal to the death receptor-signaling pathway. Given that overexpression of DEDD or FLAME-3 did not have a significant potentiation effect on death receptor induced apoptosis, the significance of the interaction with cFLIP, which is an antagonist to the death receptor pathway, is not clear at present.

DEDD and FLAME-3 were found to interact with the hTFIIIC102 subunit of the TFIIIC transcription factor by the yeast two hybrid analysis, in vitro GST-pull down and in vivo. Given the nuclear localization of the DEDD proteins, this finding suggests that these proteins could be important regulators of the TFIIIC transcriptional complex. hTFIIIC102 is an important component of the TFIIIC transcription machinery. ${ }^{23-25}$ hTFIIIC102 is one of the five hitherto identified subunits of transcription factor hTFIIIC2 which, in association with TFIIIB, is responsible for the initiation of RNA polymerase III mediated transcription of group III genes in eukaryotes. The hTFIIIC102 is a 886 amino acid long protein with distinct domains. The $\mathrm{N}$-terminal region of hTFIIIC102 has nine TPR motifs and these domains have been shown to interact with the hTFIIIB90, TBP and hTFIIIC63. ${ }^{23}$ Thus the TFIIIC102 subunit is important for recruitment of RNA polymerase III via TFIIIB interaction and for stable complex formation.

Previous studies have shown that TFIIIC is located in both the cytoplasm and nucleus. ${ }^{27,28}$ Endogenous DEDD has also been found to be mainly localized to the cytoplasm and is translocated to the nucleus upon stimulation of CD95/Fas. ${ }^{16}$ Our finding that co-expression of hTFIIIC102 with either DEDD or FLAME-3 appears to induce translocation of hTFIIIC102 to the nucleus, suggests that the DEDD proteins may regulate hTFIIIC102 transport across the nuclear membrane under normal physiological circumstances. Thus, the DEDD and FLAME-3 proteins could function as chaperones for the hTFIIIC102 to translocate it across the nuclear membrane into the nucleus to be recruited to the TFIIIC transcription complex.

The ability of DEDD proteins to inhibit transcription (our data and ref ${ }^{16,17}$ ) when overexpressed in mammalian cells, suggest that their interaction with and sequestration of hTFIIIC102 could be the reason for this inhibition. This could also explain the inhibition of $\mathrm{NF}-\kappa \mathrm{B}$ regulated gene expression by the overexpressed DEDD and FLAME-3 proteins. This inhibition might also be the reason for their observed weak apoptotic activity. It is noteworthy to mention that there are examples of association of apoptosis related proteins with transcription factors. ${ }^{29}$ BAG-1, an antiapoptotic protein, interacts with a number of nuclear hormone receptors including receptors for glucocorticoid, estrogen and thyroid hormone. ${ }^{29}$ BAG-1 can regulate retinoid activities through its interaction with $R A R$ and suggests that elevated levels of BAG-1 could potentially contribute to retinoid resistance in cancer cells. ${ }^{29}$ Thus, it would be interesting in the future to fully characterize the biological significance of the interaction between the DEDD and FLAME-3 proteins and hTFIIIC102, and whether any of these proteins might be de-regulated in cancer cells.

\section{Materials and Methods}

\section{Identification and cloning of FLAME-3}

ESTs encoding human and mouse FLAME-3 were identified by searching the National Center for Biotechnology Information GenBank expressed-sequence tags (EST) database, for sequences homologous to DEDD. The EST clones (human Genbank accession \# AA090354) and (mouse Genbank accession \# AA511237) were found to have significant homology to DEDD, but were truncated at the $5^{\prime}$ regions. These clones were obtained from the IMAGE consortium, and their entire nucleotide sequences were determined by automated sequencing. The human and mouse FLAME-3 5'-coding regions were obtained by RACE, and their full-length open reading frames were generated by overlapping PCR.

\section{Expression vectors}

Constructs encoding full length DEDD, FLAME-3, TFIIIC102 and TFIIIC102-s were generated by PCR using modified complementary PCR adapter-primers. Flag- and T7- epitope tagging was done by cloning the PCR generated $c D N A s$ of the respective genes in-frame into pFLAG CMV-2 (IBI Kodak), and pcDNA3 (Invitrogen) with T7-tag vectors, respectively. Plasmids encoding GFP fusions were constructed using pEGFP-N1 and pEGF-C1 (CLONTECH). The expression constructs for T7-tagged Flame-1/cFLIP, caspase-8, caspase-10 and FADD were described previously. ${ }^{30,31}$ Full length DEDD and FLAME-3 were overexpressed in E. coli strain DH5 $\alpha$ as N-terminally GST-fusion proteins using a pGEX vector (Pharmacia).

\section{Northern blot analysis}

Tissue distribution analysis of human FLAME-3 and TFIIIC102 mRNA was performed by Northern blot analysis on normal and tumor MTN Blots (purchased from CLONTECH). Each lane contains $2 \mu \mathrm{g}$ of 
poly $(A)^{+}$RNA from specific tissues or cell lines. The blots were probed with a radiolabeled riboprobe prepared from a full-length human FLAME-3 or TFIIIC102 cDNA template and then subjected to autoradiography.

\section{Cell culture}

293T and MCF7 cells were maintained at $37^{\circ} \mathrm{C}$ in a humidified atmosphere of $95 \%$ air and 5\% carbon dioxide in either Dulbecco's modified Eagle's medium (DMEM)/F12 or RPMI1640 (Life Technologies, Inc) containing $10 \%$ fetal bovine serum, $200 \mathrm{mg} / \mathrm{ml}$ penicillin and $100 \mathrm{mg} / \mathrm{ml}$ streptomycin sulfate.

\section{Transfection, immunoprecipitation, and Western blot analysis}

Transient transfection of $293 \mathrm{~T}$ cells was performed as described previously. ${ }^{32}$ In brief, cells were transfected with the indicated Flagtagged and T7-tagged expression plasmids ( $8 \mu \mathrm{g} / 10 \mathrm{~cm}$ plate) using the LipofectAMINE (Life Technologies, Inc.). Cell lysates were prepared in lysis buffer $(50 \mathrm{mM}$ Tris- $\mathrm{HCl}, \mathrm{pH} 7.6,150 \mathrm{mM} \mathrm{NaCl}$, $0.1 \%$ NP-40 and freshly added $1 \mathrm{mM}$ PMSF) $24-30 \mathrm{~h}$ after transfection, and immunoprecipitated with anti-FLAG mAb M5. The precipitates were resolved by SDS polyacrylamide gel electrophoresis and analyzed by immunoblotting with anti-T7 antibody, to monitor the expression level of the Flag- and T7-tagged proteins. Western blot analyses were performed on total cellular extracts with either anti-Flag M5 or anti-T7 antibodies.

\section{Fluorescence microscopy}

MCF-7-Fas cells were grown on glass coverslips in 12 well plates. Cells were transfected $24 \mathrm{~h}$ later, with constructs encoding GFP fusion proteins. Twenty-four hours after transfection, the cells were fixed with $4 \%$ paraformaldehyde in PBS for $10 \mathrm{~min}$ at room temperature. After washing twice with PBS, the coverslips were mounted on a glass slide and the fluorescence of GFP was detected by Fluorescent or confocal microscopy using excitation wavelength of $488 \mathrm{~nm}$ and detection wavelength of $522 \mathrm{~nm}$.

\section{Yeast two-hybrid screening}

The human brain cDNA matchmaker libraries in pJG4-5 (TRP1, $2 \mu \mathrm{m}$, $A p^{r}$ ) were purchased from Clontech. The yeast two-hybrid screens were performed according to the manufacturer's procedures. In brief, the fulllength DEDD open reading frame was cloned in pEG202 (HIS3, $2 \mu \mathrm{m}$, $A p^{r}$ ) in-frame with Lex A expression sequence. The PSH18-34 LacZ reporter plasmid (URA3, $2 \mu \mathrm{m}, \mathrm{Ap}^{r}$ ) was used for the interaction assays. LEU 2-selection yeast strain EGY-48 (MAT/, trp1, his3, ura3, 6opsLEU2) was used as the host in the interaction assays. The three vector primary transformants were plated onto Gal/Raff/CM, -Ura, -His, -Trp, -Leu plates and incubated for 5 days at $30^{\circ} \mathrm{C}$. All positive growing colonies were further screened on X-gal plates to test for $\beta$-galactosidase expression. The cDNA inserts in the positive library clones were sequenced by using vector specific primer. The interacting clones were further confirmed by in vitro and in vivo interaction studies.

\section{In vitro interaction assay}

The GST - DEDD and GST - FLAME-3 fusion proteins were expressed in bacteria and then bound to glutathione-Sepharose beads. ${ }^{35} \mathrm{~S}$ labeled DED-containing proteins were generated by in vitro translation with TNT reticulocyte lysates (promega). T7-hTFIIIC102 was generated by transfecting an expression construct encoding T7hTFIIIC102 into 293T cells. Extracts were prepared, $24 \mathrm{~h}$ later, from the transfected cells in a GST-binding buffer (20 mM HEPES pH 7.4, $1.5 \mathrm{mM} \mathrm{MgCl}_{2}, 100 \mathrm{mM} \mathrm{NaCl}, 1 \%$ Triton and freshly added $1 \mathrm{mM}$ DTT and $1 \mathrm{mM}$ PMSF). The ${ }^{35} \mathrm{~S}$-labeled lysates or the 293 extracts were pre-cleared by mixing with glutathione-Sepharose beads and subjected to centrifugation. The supernatants were incubated with GST-DEDD or GST-FLAME-3 immobilized on glutathione Sepharose beads for $2 \mathrm{~h}$ at $4^{\circ} \mathrm{C}$. After extensive washing, the interacting proteins were recovered by boiling the beads in SDS sample buffer and analyzed on $12 \%$ SDS-PAGE. The ${ }^{35}$ S-labeled proteins were visualized by autoradiography, while the T7-tagged hTFIIIC102 was visualized by immunoblotting with anti-T7.

\section{NF- $\kappa$ B reporter gene assay}

For transient reporter gene assay, 293T cells were seeded in 12-well tissue culture plates, and the following day the cells were transfected with $5 \mathrm{X}_{\kappa} \mathrm{B}$-luciferase reporter and $\mathrm{pRSC}-$ LacZ plasmids and expression plasmids for DEDD or FLAME-3 using the LipofectAMI$\mathrm{NE}^{\mathrm{TM}}$ method as per the manufacturer's instruction. Twenty-four hours after transfection, cells were treated with hTNF- $\alpha$ for $5 \mathrm{~h}$ prior to harvesting, and then harvested and subjected to luciferase assay as described in Lin et al. ${ }^{33}$ To normalize for transfection efficiency, all lysates were assayed for $\beta$-galactosidase activity. Luciferase activity was determined using luciferase assay system (Promega) and a FB15 luminometer (Zylux Corporation, USA). Data represent the average of at least three different individual experiments.

\section{Apoptosis assays}

The ability of FLAME-3 to induce apoptosis was assayed by transfecting human MCF-7-Fas cells $\left(1 \times 10^{5}\right.$ cells/well) in 6-well plates with $0.4 \mu \mathrm{g}$ of pEGFP-N1 reporter plasmid (Clontech), $1.0 \mu \mathrm{g}$ of empty vector or constructs encoding FLAME-3, FADD or procaspases8 using the LipofectAMINE ${ }^{\text {TM }}$ method. Cells were stained with propidium iodide and DAPI stains. Normal and apoptotic GFPexpressing cells were counted after 24,48 and $72 \mathrm{~h}$ after transfection using fluorescence microscopy. The percentage of apoptotic cells in each experiment was expressed as the mean percentage of apoptotic cells as a fraction of the total number of GFP expressing cells. The ability of DEDD or FLAME-3 to potentiate TRAIL-, TNF- $\alpha$-, or Fasinduced apoptosis was assayed by transfecting human MCF-7 cells $\left(1 \times 10^{5}\right.$ cells/well) in 6 -well plates with $1.2 \mu \mathrm{g}$ of empty vector, or DEDD or FLAME-3 expression construct plus $0.3 \mu \mathrm{g}$ pEGFP-N1 plasmid as above. Twenty-four hours after transfection cells were treated with TNF $(10 \mathrm{ng} / \mathrm{ml})$, TRAIL $(1 \mu \mathrm{g} / \mathrm{ml})$ or $\mathrm{CH}-11$ antibody $(100 \mathrm{ng} / \mathrm{ml})$ for $5 \mathrm{~h}$ and the percentages of GFP-positive, DAPIpositive apoptotic cells were determined as above.

\section{Accession numbers}

The nucleotide sequence(s) reported here has been submitted to the GenBank ${ }^{T M} / E B I$ Data Bank (accession numbers AF457575, AF457576 and AF465407).

\section{Acknowledgements}

This work was supported by National Institutes of Health Grants CA85421 and AG14357 (to ES Alnemri). SM Srinivasula is a Special Fellow of the Leukemia and Lymphoma Society. 


\section{References}

1. Ashkenazi A and Dixit VM (1998) Death receptors: signaling and modulation Science 281: 1305-1308

2. Weber $\mathrm{CH}$ and Vincenz $\mathrm{C}$ (2001) The death domain superfamily: a tale of two interfaces? Trends Biochem. Sci. 26, 475-481

3. Boldin MP, Mett IL, Varfolomeev EE, Chumakov I, Shemer-Avni Y, Camonis JH and Wallach D (1995) Self-association of the 'death domains' of the p55 tumor necrosis factor (TNF) receptor and Fas/APO1 prompts signaling for TNF and Fas/APO1 effects. J. Biol. Chem., 270, 387-391

4. Chinnaiyan AM, Tepper CG, Seldin MF, O'Rourke K, Kischkel FC, Hellbardt S, Krammer PH, Peter ME and Dixit VM (1996) FADD/MORT1 is a common mediator of CD95 (Fas/APO-1) and tumor necrosis factor receptor-induced apoptosis. J. Biol. Chem., 271, 4961-4965

5. Salvesen GS and Dixit VM (1999) Caspase activation: the induced-proximity model. Proc. Natl. Acad. Sci. USA, 96, $10964-10967$

6. Medema JP, Scaffidi C, Kischkel FC, Shevchenko A, Mann M, Krammer PH and Peter ME (1997) FLICE is activated by association with the CD95 death-inducing signaling complex (DISC). EMBO J., 16, 2794-2804

7. Yang X, Chang HY and Baltimore D (1998) Autoproteolytic activation of procaspases by oligomerization. Mol. Cell., 1, 319-325

8. Budihardjo I, Oliver H, Lutter M, Luo X and Wang X (1999) Biochemical pathways of caspase activation during apoptosis. Annu. Rev. Cell. Dev. Biol., 15, 269-290

9. Yin XM (2000) Bid, a critical mediator for apoptosis induced by the activation of Fas/TNF-R1 death receptors in hepatocytes. J. Mol. Med., 78, 203-211

10. Krueger A, Baumann S, Krammer PH and Kirchhoff S (2001) FLICE-inhibitory proteins: regulators of death receptor-mediated apoptosis. Mol. Cell. Biol., 21, $8247-8254$

11. Yeh WC, Itie A, Elia AJ, Ng M, Shu HB, Wakeham A, Mirtsos C, Suzuki N, Bonnard M, Goeddel DV and Mak TW (2000) Requirement for Casper (c-FLIP) in regulation of death receptor-induced apoptosis and embryonic development. Immunity, 12, 633-642

12. Ramos JW, Kojima TK, Hughes PE, Fenczik CA and Ginsberg MH (1998) The death effector domain of PEA-15 is involved in its regulation of integrin activation. J. Biol. Chem., 273, 33897-33900

13. Ramos JW, Hughes PE, Renshaw MW, Schwartz MA, Formstecher E, Chneiweiss $\mathrm{H}$ and Ginsberg MH (2000) Death effector domain protein PEA-15 potentiates Ras activation of extracellular signal receptor-activated kinase by an adhesion- independent mechanism. Mol. Biol. Cell., 11, 2863-2872

14. Zhang Y, Redina O, Altshuller YM, Yamazaki M, Ramos J, Chneiweiss $\mathrm{H}$, Kanaho Y and Frohman MA. (2000) Regulation of expression of phospholipase D1 and D2 by PEA-15, a novel protein that interacts with them. J. Biol. Chem., $275,35224-35232$

15. Condorelli G, Vigliotta G, lavarone C, Caruso M, Tocchetti CG, Andreozzi F, Cafieri A, Tecce MF, Formisano P, Beguinot L and BeguinotF. (1998) PED/PEA15 gene controls glucose transport and is overexpressed in type 2 diabetes mellitus. EMBO J., 17, 3858-3866

16. Stegh AH, Schickling O, Ehret A, Scaffidi C, Peterhansel C, Hofmann TG, Grummt I, Krammer PH and Peter ME. (1998) DEDD, a novel death effector domain-containing protein, targeted to the nucleolus. EMBO J., 17, 5974-5986

17. Schickling O, Stegh AH, Byrd J and Peter ME (2001) Nuclear localization of DEDD leads to caspase-6 activation through its death effector domain and inhibition of RNA polymerase I dependent transcription. Cell. Death. Differ., 8, $1157-1168$
18. Chaudhary PM, Eby MT, Jasmin A, Kumar A, Liu L and Hood L (2000) Activation of the NF-kappaB pathway by caspase 8 and its homologs. Oncogene, 19, $4451-4460$

19. Chaudhary PM, Jasmin A, Eby MT and Hood L (1999) Modulation of the NFkappa $B$ pathway by virally encoded death effector domains-containing proteins. Oncogene, 18, 5738-5746

20. Hu WH, Johnson $\mathrm{H}$ and Shu HB (2000) Activation of NF-kappaB by FADD, Casper, and caspase-8. J. Biol. Chem., 275, 10838-10844

21. Zvalova D, Formstecher E, Fauquet M, Canton B and Chneiweiss H (2001) Keeping TNF-induced apoptosis under control in astrocytes: PEA-15 as a 'double key' on caspase-dependent and MAP-kinase-dependent pathways. Prog. Brain Res., 132, 455-467

22. Leo CP, Hsu SY, McGee EA, Salanova M and Hsueh AJ (1998) DEFT, a novel death effector domain-containing molecule predominantly expressed in testicular germ cells. Endocrinology, 139, 4839-4848

23. Hsieh YJ, Wang Z, Kovelman R and Roeder RG (1999) Cloning and characterization of two evolutionarily conserved subunits (TFIIIC102 and TFIIIC63) of human TFIIIC and their involvement in functional interactions with TFIIIB and RNA polymerase III. Mol. Cell. Biol., 19, 4944-4952

24. Huang $Y$ and Maraia RJ (2001) Comparison of the RNA polymerase III transcription machinery in Schizosaccharomyces pombe, Saccharomyces cerevisiae and human. Nucleic Acids Res., 29, 2675-2690

25. Geiduschek EP and Kassavetis GA (2001) The RNA polymerase III transcription apparatus. J. Mol. Biol., 310, 1-26

26. Roth W, Stenner-Liewen F, Pawlowski K, Godzik A and Reed JC (2002) Identification and characterization of DEDD2, a death effector domaincontaining protein. J. Biol. Chem., 272, 7501-7508

27. Van Dyke MW and Roeder RG (1987) Two forms of transcription factor TFIIIC in extracts from HeLa cells. Nucleic Acids Res., 15, 5031-5039

28 Schneider HR, Waldschmidt R, Jahn D and Seifart KH (1989) Purification of human transcription factor IIIC and its binding to the gene for ribosomal 5 S RNA. Nucleic Acids Res., 17, 5003-5016

29. LiuR, Takayama S, Zheng Y, Froesch B, Chen GQ, Zhang X, Reed JC and Zhang XK (1998) Interaction of BAG-1 with retinoic acid receptor and its inhibition of retinoic acid-induced apoptosis in cancer cells. J. Biol. Chem., 273, 1698516992

30. Srinivasula SM, Ahmad M, Ottilie S, Bullrich F, Banks S, Wang Y, FernandesAlnemri T, Croce CM, Litwack G, Tomaselli KJ, Armstrong RC and Alnemri ES (1997) FLAME-1, a novel FADD-like anti-apoptotic molecule that regulates Fas/ TNFR1-induced apoptosis. J. Biol. Chem., 272, 18542-18545

31. MacFarlane M, Ahmad M, Srinivasula SM, Fernandes-Alnemri T, Cohen GM and Alnemri ES (1997) Identification and molecular cloning of two novel receptors for the cytotoxic ligand TRAIL. J. Biol. Chem., 272, 25417-25420

32. Srinivasula SM, Ahmad M, Lin JH, Poyet JL, Fernandes-Alnemri T, Tsichlis PN and Alnemri ES (1999) CLAP, a novel caspase recruitment domain-containing protein in the tumor necrosis factor receptor pathway, regulates NF-kappaB activation and apoptosis. J. Biol. Chem., 274, 17946-17954

33. Lin X, MuY, Cunningham JrET, Marcu KB, Geleziunas Rand Greene WC (1998) Molecular determinants of NF-kappaB-inducing kinase action. Mol. Cell. Biol., $18,5899-5907$ 